\title{
Knowledge Inferencing Using Artificial Bee Colony and Rough Set for Diagnosis of Hepatitis Disease
}

\author{
Kauser Ahmed P., VIT, Vellore, India \\ Debi Prasanna Acharjya, VIT, Vellore, India \\ iD https://orcid.org/0000-0003-3828-2050
}

\begin{abstract}
Vast volumes of raw data are generated from the digital world each day. Acquiring useful information and chief features from this data is challenging, and it has become a prime area of current research. Another crucial area is knowledge inferencing. Much research has been carried out in both directions. Swarm intelligence is used for feature selection whereas for knowledge inferencing either fuzzy or rough computing is widely used. Hybridization of intelligent and swarm intelligence techniques are booming recently. In this research work, the authors hybridize both artificial bee colony and rough set. At the initial phase, they employ an artificial bee colony to find the chief features. Further, these main features are analyzed using rough set generating rules. The proposed model indeed helps to diagnose a disease carefully. An empirical analysis is carried out on hepatitis dataset. In addition, a comparative study is also presented. The analysis shows the viability of the proposed model.
\end{abstract}

\section{KEYWORDS}

Approximation, Classification, Feature Selection, Modification Rate, Prediction, Reduct, Rough Set, Rule Generation

\section{INTRODUCTION}

Information and communication technology and usage of the internet is the prime factor for the massive amount of data generated each day. These accumulated data is useless unless being analyzed to get some meaningful information. Processing of these data to get meaningful information is data analytics. Additionally identifying features affecting decisions is of challenging. Machine learning techniques are used to customize and supervise data to discover useful knowledge, rules and chief factors. The extracted knowledge and rules must be accurate, readable, comprehensible, and ease of understanding. Feature selection is a primary issue in machine learning, data mining and pattern recognition to preserve the meaning of information about a given problem. Additionally, it is imperative and aims to find the minimal subset of the original features. Simultaneously, it is a crucial pre-processing technique useful for data analysis, where only a subset from the original data features is chosen by to eliminate noisy, irrelevant or redundant features (Schiezaro \& Pednini, 2013). These techniques are applied to all types of datasets to predict the main features and are an essential step used in classification, cluster analysis, and image retrieval. 
Besides, data in healthcare industries is growing exponentially. Much research has been carried out in many directions such as context-aware remote e-healthcare (Sharma \& Kumar, 2019a), service level agreement and energy cooperative cyber physical system (Sharma \& Kumar, 2019b, Sharma et al, 2019, Sharma \& Kumar 2019c), and multimedia healthcare data processing (Rathee et al, 2019). Similarly much work has been carried out in feature selection and disease diagnosis. Many algorithms about feature selection have been proposed (Das \& Liu, 1997; Jain \& Zongker, 1997; Kohavi \& John, 1997; Guyon \& Elisseeff, 2003). Recently, evolutionary and swarm intelligence algorithms are employed to optimize feature selection combination is very important. With an optimal feature subset, it is possible to gain good prediction accuracies with low computational complexity (Palanisamy \& Kanmani, 2012). A survey on six biologically inspired swarm algorithms, namely particle swarm optimization (PSO), ant colony optimization (ACO), artificial fish swarm algorithms (AFSA), artificial bee colony algorithms (ABC), firefly algorithms (FA) and bat algorithm (BA) and its application in feature selection is also carried out (bin \& binti, 2014). Schiezaro \& Pednini (2013) model the ABC algorithm for feature selection and classified different data sets. The model achieved a higher classification accuracy of $87.10 \%$ on reducing the number of features for hepatitis dataset and $98.26 \%$ for labour dataset (Forsati, Moayedikia \& Keikha, 2012). It is experimented over a benchmark data sets and demonstrate that applying ABC in feature selection is a feasible method. Palanisamy \& Kanmani (2012) have discussed ABC approach for optimizing feature selection. The model reduces feature size and improves in classification accuracy with low computational complexity (Shokouhifar \& Sabet, 2010; Uzer, Yilmaz \& Inan, 2014).

Further, two major conflicting objectives of artificial bee colony optimization on feature selection is also discussed in the literature (Hancer et al, 2018). In 2019, an improved artificial bee colony technique based on elite strategy and dimension learning is introduced (Xiao et al, 2019). The improved binary artificial bee colony algorithm is analyzed over microarray data for feature selection (Wang \& Dong, 2019). Similarly, an integrated model integrating artificial bee colony and gradient boosting decision tree is carried out for feature selection (Rao et.al, 2019). Furthermore with new versions of onlooker bee, natural selection methods for artificial bee colony is introduced for feature selection (Awadallah et al, 2019). But, all these feature selection methods fails to handle uncertainties. Simultaneously, rule generation while analysing real life problems is not addressed.

On the other hand, the rough set of Pawlak (1982), established as a sound theory to resolve problems related to uncertainty, incomplete information and imprecise variety of applications. Shu \& Shen (2014) addressed an incremental approach based on rough set for feature selection, and it has been applied to incomplete dynamic data. Further, the rough set has extended to fuzzy rough set (Dubois \& Prade, 1990), rough set on fuzzy approximation space (Acharjya \& Tripathy, 2008), rough set on intuitionistic fuzzy approximation space (Acharjya \& Tripathy, 2007), rough set on two universal sets (Tripathy \& Acharjya, 2013), intuitionistic fuzzy rough set on two universal sets (Acharjya \& Tripathy, 2012), etc. for feature selection and knowledge acquisition. Also, the rough set is hybridized with formal concept analysis (Tripathy, Acharjya \& Cynthya, 2013), rough set on fuzzy approximation space in hybridized with the soft set (Das \& Acharjya, 2014), and knowledge acquisition is carried out. However, the optimization in feature selection is not being carried out through a reduction in attributes is being done. Therefore, it is essential to think of optimized swarm intelligence techniques that can minimize the number of attributes. Besides, the major drawback of rough computing is that it generates too many rules. Sometimes it is difficult to infer rules that are helpful for the problem taken into consideration (Saxena, Gavel \& Shrivas, 2014; Swiniarski \& Skowron, 2003; Zhong, Dong \& Ohsuga, 2001).

Taking into account the above limitations, in this paper, we hybridize rough set with artificial bee colony. The prime objective of hybridization is to keep main attributes while optimizing features and inferencing rules. In the early stage, we employ the artificial bee colony to identify optimized features. Further these optimized features are used to generate rules using the rough set. Finally, we 
compare both the rough set model and the hybridized model that integrates both artificial bee colony and rough set (ABCRS) regarding rule generation.

The article is articulated as follows. Section 2 talks about foundations of artificial bee colony and the optimal procedure of obtaining best features. Following section 2, fundamental of rough set is explored in section 3 to unfold the article. Explanation of the proposed model is discussed in section 4. An empirical study on hepatitis disease is presented in section 5. Further result and analysis of the empirical study and a comparative analysis with rough set model is also carried out. The article is concluded in section 6 .

\section{FOUNDATIONS OF ARTIFICIAL BEE COLONY}

The proposed model of knowledge inferencing uses artificial bee colony in its first phase for feature selection. Feature selection finds the feasible subset of features from an information system. Traditional optimization algorithms are inefficient in selecting feasible subset of features from an information system because feature selection is a non-deterministic polynomial complete problem. Therefore, meta-heuristic algorithms are used for identifying feasible set of features. Much research has been carried out using bio-inspired computing algorithms (Kaur \& Goyal, 2011). In this proposed model, we use artificial bee colony (ABC) algorithm for obtaining a feasible set of features without loss of any knowledge. Now, we state briefly the concepts and notations regarding artificial bee colony algorithm.

Artificial bee colony algorithm is a meta-heuristic algorithm for solving optimizing numerical problem (Karaboga, 2008). The fundamental concept behind the algorithm is that it simulates the foraging behaviour of honey bees. The algorithm has three phases such as employed bee, onlooker bee and scout bee. In the first two phases, bees exploit the sources by local searches in the neighborhood of the solutions selected. The employed bee phase follows deterministic selection whereas the probabilistic selection is followed in the onlooker bee phase. In scout bee phase, solutions that are not beneficial anymore for search progress are abandoned and new solutions are inserted instead of them to explore new regions in the search space. It is observed that, the algorithm has a wellbalanced exploration and exploitation ability. The following algorithm (Bao \& Zeng, 2009) provides the general view of the artificial bee colony technique. The flow diagram of artificial bee colony for feature selection is depicted in Figure 1.

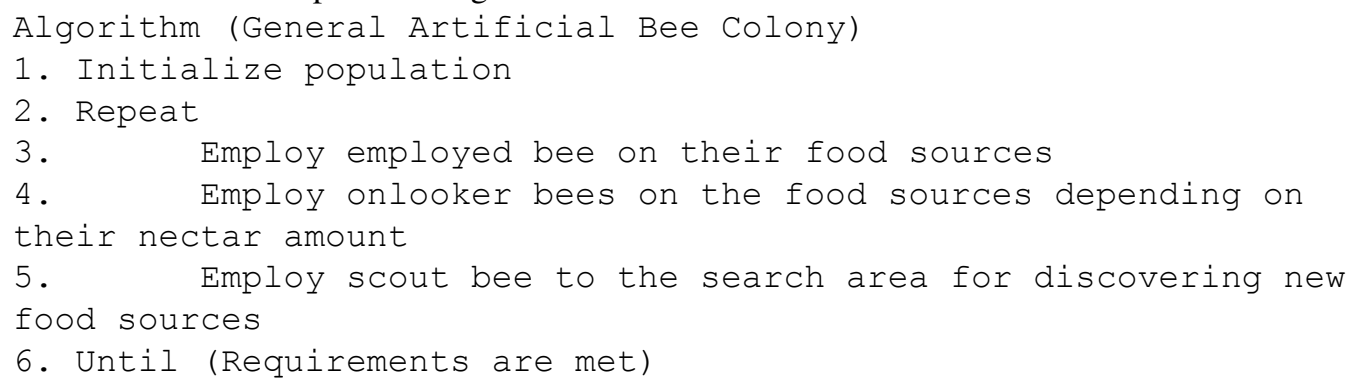

The artificial bee colony algorithm (Karaboga \& Basturk, 2008; Karaboga \& Celal, 2011; Karaboga \& Akay, 2009) starts with initialization of food sources. The initial food source can be created randomly in such a manner that each of them must be a possible solution. The initial food source can be determined using equation (1), where $i=1,2, \cdots, n$ refers number of objects, $j=1,2, \cdots, d$ is the number of features to be optimized, and $\operatorname{rand}(0,1)$ generates a random values between 0 and 1 .

$$
q_{i j}=q_{j}^{\min }+\operatorname{rand}(0,1) \times\left(q_{j}^{\max }-q_{j}^{\min }\right)
$$


Figure 1. Flow diagram of artificial bee colony for feature selection

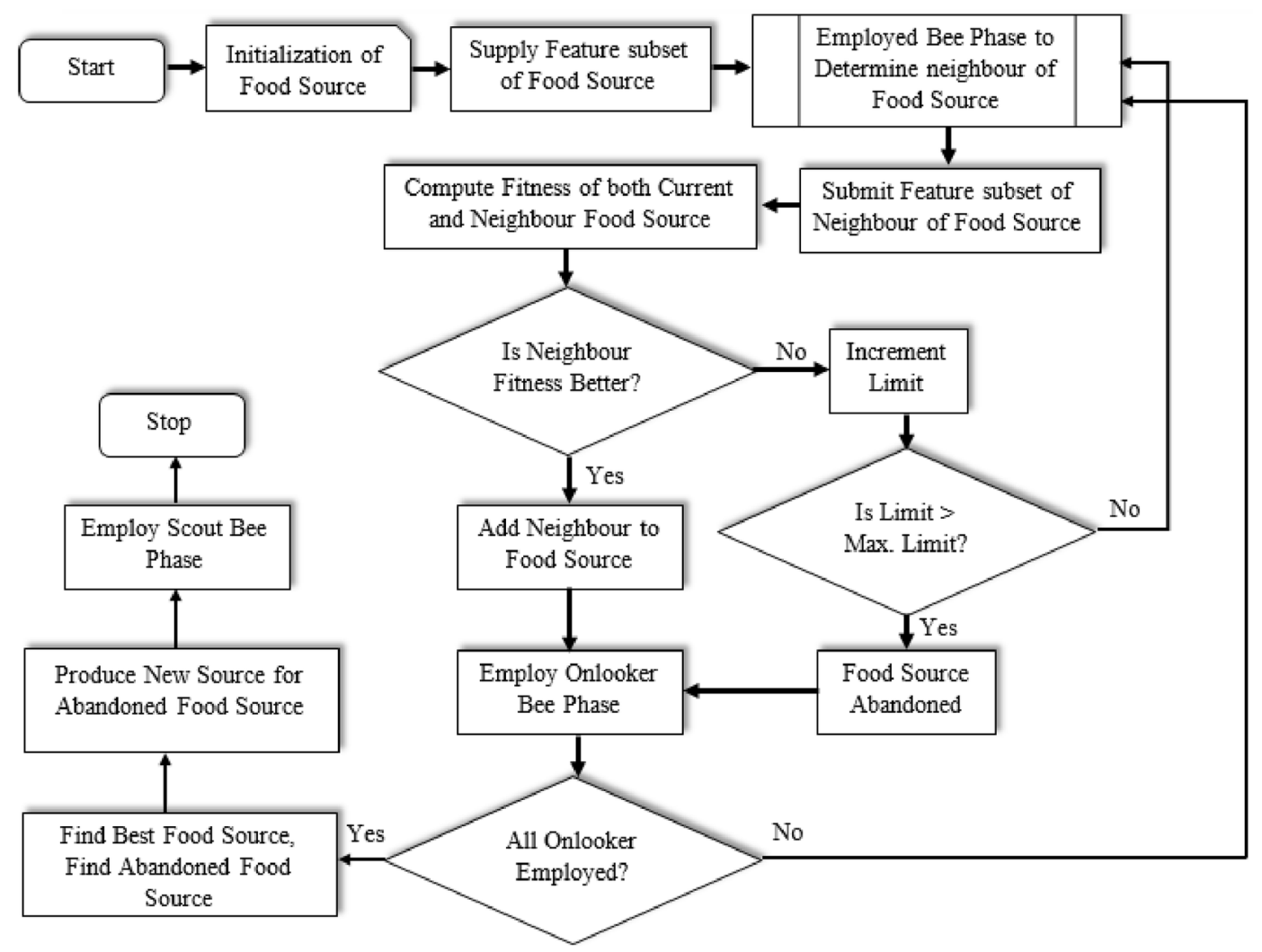

In employed bee phase, every employed bee search a new food source (solution) in their neighbourhood using equation (2), where for every food source $q_{i}$, a new food source $v_{i}$ is identified by modifying optimization feature $j$. The indices $j, k$ are selected randomly other than $i$, and $\phi_{i j}$ is a real number between -1 and 1 .

$v_{i j}=q_{i j}+\phi_{i j} \times\left(q_{i j}-q_{k j}\right)$

After a new food source $v_{i}$ is identified, fitness ( fit ) is computed for every food source using equation (3), where $f_{i}$ is the cost function.

fit $_{i}= \begin{cases}\frac{1}{1+f_{i}} & \text { if } f_{i} \geq 0 \\ 1+\left|f_{i}\right| & \text { if } f_{i}<0\end{cases}$

Further compute the probability $\left(p_{i}\right)$ of the $i^{\text {th }}$ food source using their fitness and probability function defined in equation (4). Using this probability value, the onlooker bees choose a food source for exploring further. 


$$
p_{i}=\frac{f i t_{i}}{\sum_{i=1}^{n} f i t_{i}}
$$

The neighborhood of the food source is further explored like employed bee as discussed above by onlooker bees. In scout bee phase, the algorithm identifies any exhausted source to be abandoned. For this purpose, a variable 'Limit' is introduced. If the 'Limit' is greater than the 'Maximum Limit', then the food source is abandoned. Besides, a new food source is discovered by scout bee.

\section{FUNDAMENTALS OF ROUGH SET}

An information system is a two-dimensional system in which horizontal and vertical dimension refers to objects and attributes (features) respectively. It means that with every object of the universe is associated with some information and it leads to the underlying philosophy of the rough set. Same information characterized by various objects is indiscernible. The indiscernibility relation obtained using this way is the mathematical foundation to rough set theory. Rough set (Pawlak, 1982), is a mathematical approach for various purposes such as feature selection and extraction, and attributes reduction and mining decision rules. The main advantage is that it does not need any preliminary information about the data. Therefore, it classifies imprecise, uncertain or incomplete information even though it is present in data. Moreover, it helps in knowledge discovery using partition properties and the discernibility matrix.

Let $Q$ be a finite non-empty set called the universe. Suppose $R \subseteq(Q \times Q)$ is an equivalence relation on $Q$. The equivalence relation $R$ defines the attributes $P$ partitions the universe $Q$ into disjoint equivalence classes known as elementary concepts. Every union of elementary concepts is known as definable set and $(Q, R)$ is called an approximation space. Further, we can characterize a target set $Z \subseteq Q$ by a pair of lower and upper approximations $\underline{R} Z$ and $\bar{R} Z$ respectively as below.

$$
\begin{aligned}
& \underline{R} Z=\bigcup\{Y \in Q / R: Y \subseteq Z\} \\
& \bar{R} Z=\bigcup\{Y \in Q / R: Y \cap Z \neq \phi\}
\end{aligned}
$$

The $R$ - boundary of $Z, B N_{R}(Z)$ is given by $B N_{R}(Z)=(\bar{R} Z-\underline{R} Z)$. We say $Z$ is rough with respect to $R$ if and only if $\underline{R} Z \neq \bar{R} Z$ or equivalently $B N_{R}(Z) \neq \phi$. Thus, set $Z$ is rough with respect to $R$ if and only if it is not $R$-definable. The target set $Z$ is $R$-definable if $B N_{R}(Z)=\phi$.

\subsection{Core and Reduct}

One of the important concepts of the rough set is reduct and core. Reduct can minimize attributes and make object classification satisfy the full set of attributes. The object belonging to all reducts is known as the core. In general, core and reduct give the decision maker simple and easy information. If the attributes are dependent, using the dependency properties of attributes, we find all possible minimal subsets of attributes that have the same number of simple sets without loss of any knowledge in the reduced information system. To express mathematically, we need some auxiliary notations. 
Let $A \subseteq P$ and $p \in A$. The attribute $p$ is dispensable in $A$ if $Q / A=Q /(A-\{p\})$. Otherwise $p$ is indispensable in $A$. Set $A$ is independent if all of its attributes are indispensable. Reduct $A^{\prime}$ of $A$ is a subset of attributes of $A$ such that the equivalence class induced by $A$ is same as the equivalence class induced by $A^{\prime}$, i.e., $Q / A=Q / A^{\prime}$. Thus, we may have many reducts in an information system. The attributes belonging to all reducts is known as the core. we define it as $\operatorname{core}(A)=\bigcap \operatorname{Re} d(A)$.

If $P=(C \cup D)$, where $C$ represents the set of conditional attributes and $D$ represents the decisions, then the information system is termed as decision system. The positive region with respect to set of conditional attributes $C$ is defined as $P O S_{C}(D)$, where

$P O S_{C}(D)=\bigcup_{X \in\{Q / D\}} \underline{C} X$

The degree of dependency between $C$ and $D$ is given as $\gamma(C, D)$ and is defined as in Equation (7).

$\gamma(C, D)=\frac{\left|P O S_{C}(D)\right|}{|Q|}$

Let us consider a sample dengue information system as shown in Table 1. It consists of 12 objects $Q=\left\{q_{1}, q_{2}, q_{3}, \cdots, q_{12}\right\}$ with attributes $P=\left\{p_{1}, p_{2}, p_{3}, p_{4}, p_{d}\right\}$, where $p_{1}$ represents high Fever, $p_{2}$ represents headaches, $p_{3}$ represents muscle pain, and $p_{4}$ represents fatigue. The attribute $p_{d}$, represented as dengue is considered as the decision attribute.

On employing indiscernibility relation over conditional attributes $p_{1}, p_{2}, p_{3}$, and $p_{4}$ we get the following classifications. From the classification it is clear that $Q / P \neq Q /\left(P-\left\{p_{i}\right\}\right) ; i=1,2,3,4$ and hence none of the attributes are superfluous.

$$
\begin{aligned}
& Q / P=\left\{\left\{q_{1}, q_{8}\right\},\left\{q_{2}, q_{3}\right\},\left\{q_{4}, q_{6}, q_{7}\right\},\left\{q_{5}, q_{9}, q_{11}\right\},\left\{q_{10}\right\},\left\{q_{12}\right\}\right\} \\
& Q /\left(P-\left\{p_{1}\right\}\right)=\left\{\left\{q_{1}, q_{8}\right\},\left\{q_{2}\right\},\left\{q_{3}\right\},\left\{q_{4}, q_{6}\right\},\left\{q_{5}, q_{9}, q_{11}, q_{12}\right\},\left\{q_{7}\right\},\left\{q_{10}\right\}\right\} \\
& Q /\left(P-\left\{p_{2}\right\}\right)=\left\{\left\{q_{1}, q_{8}\right\},\left\{q_{2}, q_{7}\right\},\left\{q_{3}, q_{4}, q_{6}\right\},\left\{q_{5}, q_{9}, q_{11}\right\},\left\{q_{10}\right\},\left\{q_{12}\right\}\right\} \\
& Q /\left(P-\left\{p_{3}\right\}\right)=\left\{\left\{q_{1}, q_{8}\right\},\left\{q_{2}\right\},\left\{q_{3}\right\},\left\{q_{4}, q_{6}\right\},\left\{q_{5}, q_{9}, q_{11}\right\},\left\{q_{7}\right\},\left\{q_{10}, q_{12}\right\}\right\} \\
& Q /\left(P-\left\{p_{4}\right\}\right)=\left\{\left\{q_{1}, q_{8}\right\},\left\{q_{2}\right\},\left\{q_{3}\right\},\left\{q_{4}, q_{5}, q_{6}, q_{9}\right\},\left\{q_{7}\right\},\left\{q_{10}\right\},\left\{q_{11}\right\},\left\{q_{12}\right\}\right\}
\end{aligned}
$$

On considering $Z=\left\{q_{3}, q_{4}, q_{5}, q_{6}, q_{10}, q_{11} q_{12}\right\}$, we get $\underline{P} Z=\left\{q_{5}, q_{9}, q_{10}, q_{11}, q_{12}\right\}$; and $\bar{P} Z=\left\{q_{2}, q_{3}\right.$, $\left.q_{4}, q_{6}, q_{7}, q_{5}, q_{9}, q_{11}, q_{10}, q_{12}\right\}$. Figure 2 depicts the classification of objects with respect to conditional attributes. The lower, upper approximation and boundary region is also presented.

\subsection{Rule Generation}

A decision rule in a decision system is of the form $\phi \rightarrow \psi$, where $\phi$ the set of conditional parameters and $\psi$ is the decision. The various measures of decision rule are support, strength, and accuracy of the rule. The support is defined as $\operatorname{Supp}(\phi, \psi)=\operatorname{Card}(\|\phi \wedge \psi\|)$ whereas strength is defined as 
Table 1. Sample dengue information system

\begin{tabular}{|c|c|c|c|c|c|}
\hline Patients & High Feaver & Headaches & Muscle Pain & F a t i g u e & D e n g u e \\
\hline$q_{1}$ & No & No & No & Normal & No \\
\hline$q_{2}$ & Yes & No & Yes & High & No \\
\hline$q_{3}$ & Yes & Yes & Yes & High & Yes \\
\hline$q_{4}$ & No & Yes & No & High & Yes \\
\hline$q_{5}$ & No & No & No & Very High & Yes \\
\hline$q_{6}$ & No & Yes & Yes & Very High & Yes \\
\hline$q_{7}$ & Yes & No & Yes & High & No \\
\hline$q_{8}$ & No & No & No & Normal & No \\
\hline$q_{9}$ & Yes & Yes & Yes & Very High & Yes \\
\hline$q_{10}$ & Yes & Yes & No & Very High & Yes \\
\hline$q_{11}$ & No & Yes & Yes & Very High & Yes \\
\hline$q_{12}$ & Yes & Yes & Yes & Very High & Yes \\
\hline
\end{tabular}

$\sigma(\phi, \psi)=\operatorname{Supp}(\phi, \psi) / \operatorname{Card}\left(\|\phi\|_{\psi}\right)$. The accuracy of the rule is defined as in Equation (7), where $N_{\text {Supp }}(\phi, \psi)$ denotes the non-supporting objects of the rule $\phi \rightarrow \psi$.

Accuracy $=\frac{|\operatorname{Supp}(\phi, \psi)|}{\left|\operatorname{Supp}(\phi, \psi)+N_{\text {Supp }}(\phi, \psi)\right|}$

Now we briefly discuss decision rule generation procedure using rough set. The qualitative decision system is given as input and we get candidacy rules as output. The steps involved in the reduct computation and decision rule generation procedure is given below.

Algorithm (Computation of Reduct)

1. Initialize reduct, Red with a null set; Red $=\{\}$

2. Repeat

3. Assign Red to $T$; $T \leftarrow$ Red

4. For each conditional attribute of $C ; \forall X \in(C-R e d)$

5. Compute the degree of dependency with assumed reduct set 


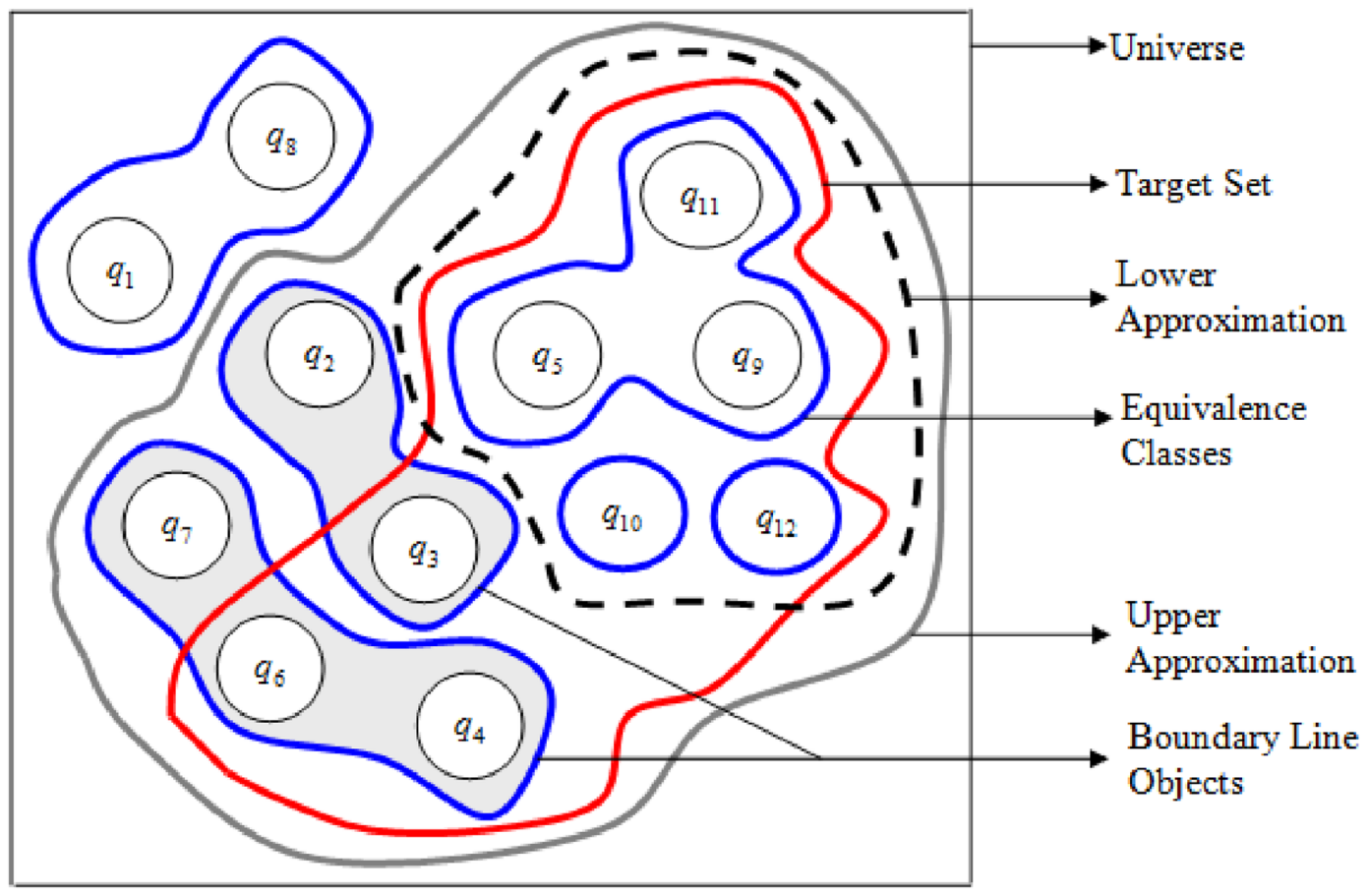

T.

6. If $\gamma_{\operatorname{Red} \cup\{X\}}(D)>\gamma_{T}(D)$

7. Then $T \leftarrow$ Red $\cup\{X\}$

8. Make $T$ as new reduct; $R e d \leftarrow T$

9. Until reduct, Red can define the decision class: $\gamma_{\text {Red }}(D)=\gamma_{C}(D)$

10. Return Red

Algorithm (Decision Rule Generation)

1. Set decision $i=1$

2. Compute a set of reducts considering all the condition

parameters for each decision.

3. Replace $i=i+1$. If all the objects have been chosen, then go

to step 4. Else go to step 5.

4. Compute the number of supporting objects of the decision rule $\operatorname{Supp}(\phi, \psi)$ after combining the identical reducts.

5. Compute the strength of each decision rule $\sigma(\phi, \psi)$.

6. Obtain the accuracy of each decision rule using Equation (8) .

7. Terminate the process and write the rules.

\subsection{A Numerical Illustration of Rough Rule Generation}

In this section, we explain the rough set rule generation procedure for the decision system presented in Table 1. The decision rules generated are given in Table 2. For example, rule 1 of the exemplary decision system presented in Table 1 can be expressed as: If fatigue $\left(p_{4}\right)$ is normal, then there is no dengue. The strength of this rule is $50 \%$ whereas its accuracy is $100 \%$. 
Table 2. Decision rules of the exemplary decision system presented in Table 1

\begin{tabular}{|c|l|c|c|c|}
\hline Rule No. & \multicolumn{1}{|c|}{ Description } & Support & Strength & Accuracy \\
\hline 1. & If $p_{4}=$ normal, then $p_{d}=$ No dengue & 2 & $50 \%$ & $100 \%$ \\
\hline 2. & If $p_{1}=$ yes and $p_{2}=$ no, then $p_{d}=$ No dengue & 2 & $50 \%$ & $100 \%$ \\
\hline 3. & If $p_{2}=$ no and $p_{3}=$ yes, then $p_{d}=$ No dengue & 2 & $50 \%$ & $100 \%$ \\
\hline 4. & If $p_{2}=$ no and $p_{4}=$ high, then $p_{d}=$ No dengue & 2 & $50 \%$ & $100 \%$ \\
\hline 5. & If $p_{2}=$ yes then $p_{d}=$ dengue is yes & 7 & $87.5 \%$ & $100 \%$ \\
\hline 6. & If $p_{4}=$ very high then $p_{d}=$ dengue is yes & 6 & $75 \%$ & $100 \%$ \\
\hline
\end{tabular}

\section{PROPOSED RESEARCH DESIGN}

This section discusses on proposed research design. The proposed research design consists of two phases. In the initial phase, the proposed model of knowledge inferencing uses artificial bee colony to find the chief features. Second phase of the model employs rough set data analysis for rule generation. The proposed research design consists of data preparation module, rule generation module, and cross validation module. The data preparation module starts from problem identification to data partition. Identification of a problem is a critical task. Once, the problem is identified, data are collected. Further data cleaning technique is employed to process these data. Data cleaning task is done to remove missing attributes values. In this research work, we employ the integrated model that integrates artificial bee colony and rough set data analysis (ABCRS) technique to inference knowledge. The model is applied to hepatitis disease to inference knowledge. After data processing, the processed data is partitioned into training dataset of 55\% and testing dataset of $45 \%$. An overview of the proposed research design is depicted in Figure 3.

The rule generation module consists of artificial bee colony algorithm $(\mathrm{ABC})$ and rough set rule generation (RS). The partitioned data is executed using artificial bee colony algorithm for identifying minimal features without impacting the diagnosis of hepatitis disease. Further, the rough set rule generation algorithm is employed to mine knowledge. The support, strength, and accuracy of each rule is obtained. The rules whose accuracy is more than the threshold of $65 \%$ are selected as candidacy rules. These candidacy rules are passed to cross validation module.

The cross validation module validates the candidacy rules obtained in training phase using 5 fold cross validation. Confusion matrix is generated to identify the true positive $(T P)$, true negative $(T N)$, false positive $(F P)$ and false negative $(F N)$ to predict precision (Prec.) and recall (Rec.). Accuracy of proposed model can be calculated in cross validation module. A confusion matrix is constructed for each rule and the accuracy (Acc.) of each rule is obtained. The precision, recall, F-measure, and accuracy are computed by using the following equations (9), (10), (11), and (12).

Precision (Prec.) $=\frac{|T P|}{|T P+F P|}$ 
International Journal of Healthcare Information Systems and Informatics

Volume 16 • Issue 2 • April-June 2021

Figure 3. Proposed research design of ABCRS model

\section{Data Preparation Module}

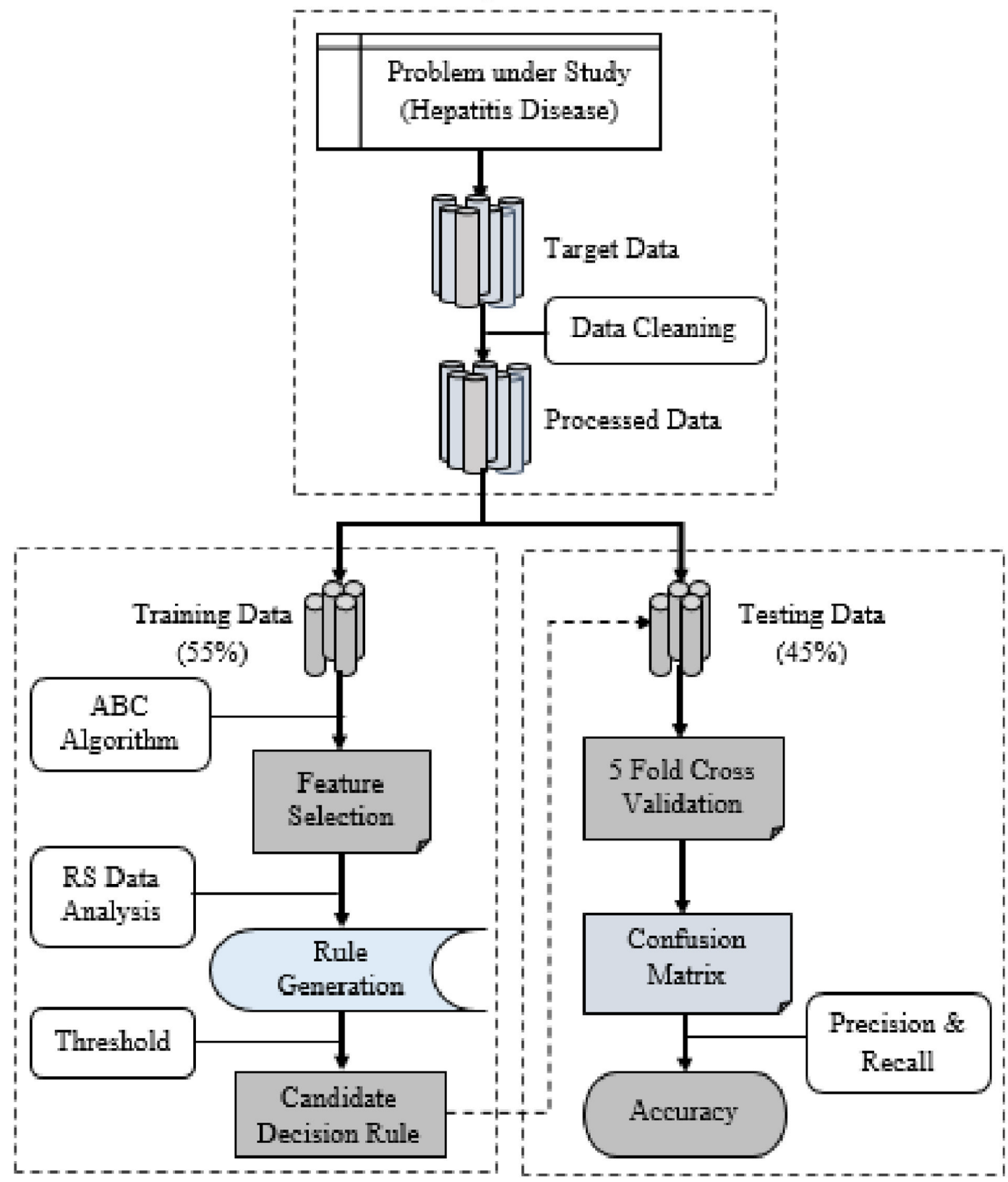

Rule Generation Module

Cross Validation Module 
Recall (Rec.) $=\frac{|T P|}{|T P+F N|}$
F-measure $=2 \times\left(\frac{\text { Prec. } \times \text { Rec. }}{\text { Prec. }+ \text { Rec. }}\right)$

$\operatorname{Accuracy}($ Acc. $)=\frac{|T P+T N|}{|T P+F P+T N+F N|}$

\section{AN EMPIRICAL STUDY ON HEPATITIS DISEASE}

This section presents an empirical study on hepatitis disease. Hepatitis disease appears in liver. Liver is an organ which has a wide range of functions, including digestion, energy production, glycogen storage, detoxification, and regulation of blood glucose. Hepatitis locates in the cells of liver tissue leading to the loss of functioning of these cells. It is of five different types such as hepatitis A, hepatitis $\mathrm{B}$, hepatitis $\mathrm{C}$, hepatitis $\mathrm{D}$, and hepatitis $\mathrm{E}$. However, hepatitis $\mathrm{B}$ is the most common liver infection in the world. It is transmitted through contact with infectious body fluids. Injection drug use, having sex with an infected partner, or using of infected persons razors may cause of hepatitis $\mathrm{B}$. The various symptoms are dark urine pale poop, loss of appetite, ascites, weakness, violation of blood clotting, headache, stomach bleeding, itching of skin, fever, and jaundice. For better visualization Figure 4

Figure 4. Various symptoms of Hepatitis B disease

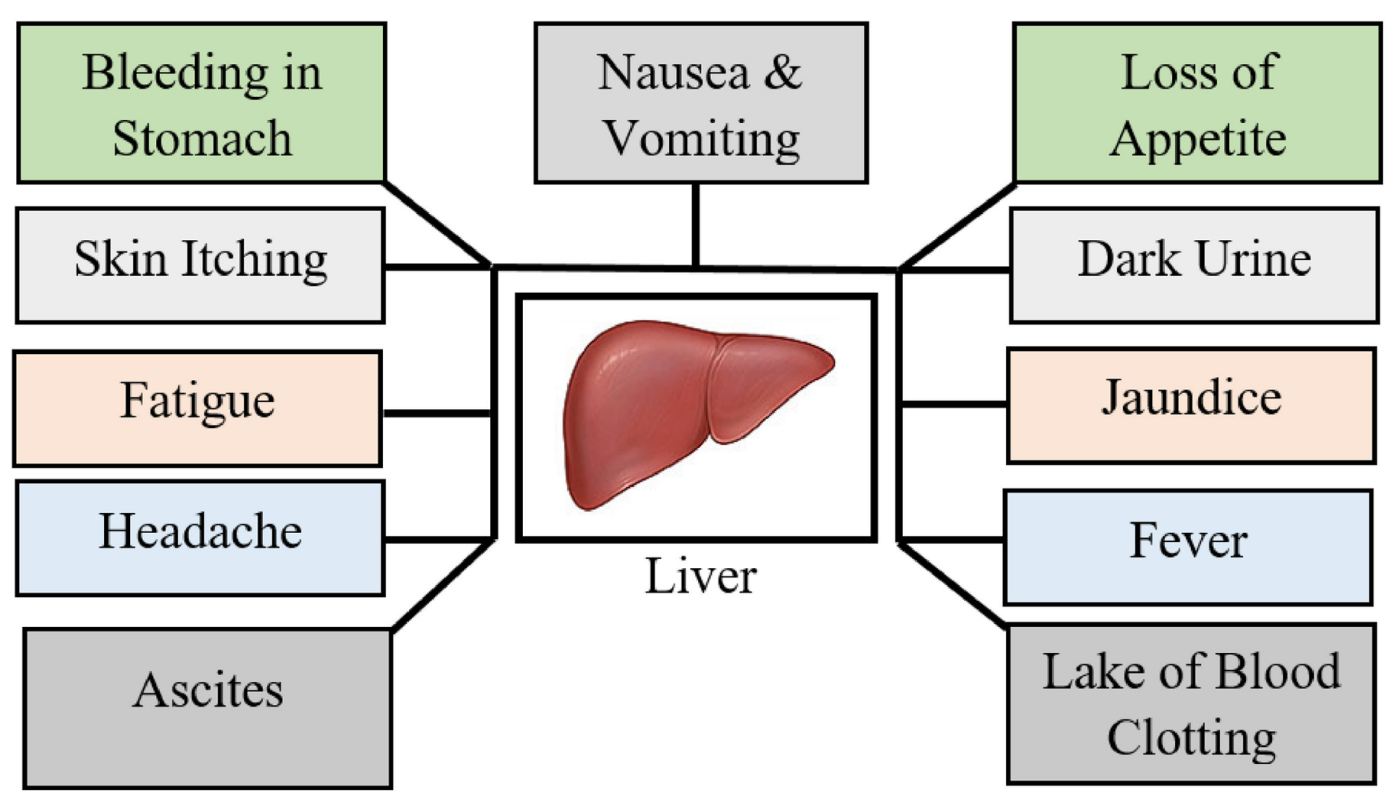


depicts the various symptoms of hepatitis B disease. An early diagnosis of these diseases saves life of a patient. Therefore, it is essential to analyze such data for early detection of it.

The classical major step towards it is feature extraction and classification. Thus, there is a need to extract the most significant features from hepatitis dataset for the diagnosis of the diseases. In this model, we use artificial bee colony to identify the most significant features. Further, in post phase we use rough set data analysis technique to find out the rules. This in deed will help us to detect the disease at an early stage.

The attributes that are considered for hepatitis disease and its range are listed in Table 3. The information on this dataset is obtained from UCI repository and some reputed hospitals of Tamilnadu, India. The dataset consists of 19 conditional attributes $\left\{p_{1}, p_{2}, \ldots, p_{19}\right\}$ and one decision attribute $\left\{p_{d}\right\}$. Further, the attributes that takes continuous values are classified according to domain experts. For example, bilirubin is classified into 4 categories such as $0.3-1.9 ; 2-3.9 ; 4-5.9$; and $6-8$. We denote these classes as low, medium, high and very high. We use symbols 1, 2, 3, 4 for low, medium, high and very high respectively while analysing the hepatitis decision system.

The hepatitis decision system collected from UCI repository contains information of 155 patients. Additionally, we have collected 237 data from reputed hospitals of Tamilnadu, India. In all together, we have 392 patients' data. It is also observed that 73 patients' data are incomplete and so discarded from the decision system. Finally, for further analysis we have considered 319 patients information. For better understanding, a sample hepatitis decision system of 10 objects is presented in Table 4 .

\subsection{Experimental Result Analysis}

The experiments were conducted with a Laptop having configuration as Intel Core i5-4200U CPU $1.60 \mathrm{GHz} 2.30 \mathrm{GHz}, 4 \mathrm{~GB}$ RAM, Windows 8 operating system, and Python. The total 319 patients' data is divided into two parts such as training data of $175(55 \%)$ and testing data of $144(45 \%)$. The training data is processed for identifying best features using artificial bee colony. After 1000 iterations it is found that 8 features whose significance values are more than the trend line are selected. For better understanding it is depicted in Figure 5. The selected features are age $\left(p_{1}\right)$, gender $\left(p_{2}\right)$, spiders $\left(p_{11}\right)$, ascites $\left(p_{12}\right)$, varices $\left(p_{13}\right)$, histology $\left(p_{14}\right)$, bilirubin $\left(p_{15}\right)$, and protime $\left(p_{19}\right)$. Further, the other features are eliminated from the training data of the information system. The reduced information system is analysed using rough set data analysis. The rules that are generated from the reduced information system are presented in Table 5. The rules whose accuracy is more than the predefined threshold value of $65 \%$ are selected as candidacy rules. These candidacy rules are further tested with testing dataset of 144 objects. Further confusion matrix is created.

From the above rules it is clear that, 4 rules such as rule 4,12,13 and 16 are having accuracy less than $65 \%$ and hence discarded from the decision rules. The rest 15 rules are further analysed with testing dataset of 144 objects to compute accuracy. The testing dataset consists of 69 live cases and 75 die cases. Further, the confusion matrix is computed and is presented in Table 6. From Table 6 , it is clear that ABCRS hybridization gives accuracy of $96.2 \%$.

\subsection{K-Fold Cross Validation}

This section computes the accuracy of the proposed model ABCRS by using K-fold cross validation. The total number of objects of testing dataset is divided into 5 folds randomly. First 4 folders contain 29 objects whereas $5^{\text {th }}$ folder contains 28 objects. For each folder we compute the precision, recall, F-measure, and accuracy. Folder 1 contains 14 live cases and 15 die cases of the dataset. Folder 2 contains 15 live cases and 14 die cases of the dataset. Folder 3 contains 16 live cases and 13 die cases. Folder 4 contains 11 live cases and 18 die cases whereas folder 5 contains 13 live cases and 15 die cases. Confusion matrix of all the folders are computed. The following Table 7 depicts the confusion matrix of Folder 1. Similarly, the confusion matrix for all the folders are computed. The 
Table 3. Attribute representation table of hepatitis disease

\begin{tabular}{|c|c|c|c|c|c|}
\hline Attribute & Range & Classification & Attribute & Range & Classification \\
\hline $\operatorname{Age}\left(p_{1}\right)$ & {$[7-78]$} & $\begin{array}{l}7-25 ; \text { Low } \\
25-45 ; \text { Medium } \\
46-65 ; \text { High } \\
\geq 66 ; \text { Very High }\end{array}$ & Gender $\left(p_{2}\right)$ & -- & $\begin{array}{ll}\text { Male } & (1) \\
\text { Female } & (0)\end{array}$ \\
\hline Steroid $\left(p_{3}\right)$ & -- & $\begin{array}{ll}\mathrm{N} \text { o } & \left(\begin{array}{l}0 \\
\mathrm{Y} \text { e s }\end{array}\right.\end{array}$ & Antivirals $\left(p_{4}\right)$ & -- & $\begin{array}{lll}\text { N o } & \left(\begin{array}{lll}0 \\
\text { Y e s }\end{array}\right. & \left(\begin{array}{l}1 \\
1\end{array}\right)\end{array}$ \\
\hline Fatigue $\left(p_{5}\right)$ & -- & $\begin{array}{ll}\mathrm{N} \text { o } & \left(\begin{array}{l}0 \\
\mathrm{Y} \text { e s }\end{array}\right.\end{array}$ & Malaise $\left(p_{6}\right)$ & -- & $\begin{array}{lll}\mathrm{N} \text { o } & & \left(\begin{array}{lll}0 & \end{array}\right) \\
\mathrm{Y} \text { e s } & & \left(\begin{array}{l}1 \\
1\end{array}\right)\end{array}$ \\
\hline Anorexia $\left(p_{7}\right)$ & -- & 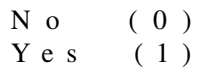 & Liver big $\left(p_{8}\right)$ & -- & $\begin{array}{ll}\text { N o } & \left(\begin{array}{ll}0 \\
\text { Y e s }\end{array}\right. \\
\text { ( } 1)\end{array}$ \\
\hline Liver firm $\left(p_{9}\right)$ & -- & $\begin{array}{ll}\mathrm{N} \text { o } & \left(\begin{array}{l}0 \\
\mathrm{Y} \text { e s }\end{array}\right.\end{array}$ & $\begin{array}{l}\text { Spleen palpable ( } \\
\left.p_{10}\right)\end{array}$ & -- & $\begin{array}{ll}\text { N o } & \left(\begin{array}{l}0 \\
\text { Y e s }\end{array}\right.\end{array}$ \\
\hline Spiders $\left(p_{11}\right)$ & -- & $\begin{array}{ll}\mathrm{N} \text { o } & \left(\begin{array}{l}0 \\
\mathrm{Y} \text { e s }\end{array}\right.\end{array}$ & Ascites $\left(p_{12}\right)$ & -- & 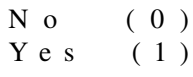 \\
\hline Varices $\left(p_{13}\right)$ & -- & $\begin{array}{ll}\mathrm{N} \text { o } & \left(\begin{array}{l}0 \\
\mathrm{Y} \text { e s }\end{array}\right.\end{array}$ & Histology $\left(p_{14}\right)$ & -- & 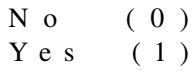 \\
\hline Bilirubin $\left(p_{15}\right)$ & $\begin{array}{lll}{\left[\begin{array}{ll}0 & .3 \\
- & 8\end{array}\right]} & -\end{array}$ & $\begin{array}{l}0.3-1.9 ; \text { Low } \\
2-3.9 ; \text { Medium } \\
4-5.9 ; \text { High } \\
6-8 ; \text { Very High }\end{array}$ & $\begin{array}{l}\text { Alk phosphate ( } \\
\left.p_{16}\right)\end{array}$ & $\begin{array}{l}{[26-} \\
295]\end{array}$ & $\begin{array}{l}26-96 ; \text { Low } \\
96.1-147 ; \\
\text { M e d i u m } \\
147.1 \quad- \\
194 ; \text { High } \\
194.1- \\
295 \text {; Very High }\end{array}$ \\
\hline $\operatorname{SGOT}\left(p_{17}\right)$ & $\begin{array}{lll}{\left[\begin{array}{lll}1 & 4 & - \\
6 & 4 & 8\end{array}\right]}\end{array}$ & $\begin{array}{l}14-98 ; \text { Low } \\
98.1-182 ; \\
\text { M e d i u m } \\
182.1 \quad- \\
278 ; \text { High } \\
278.1- \\
648 ; \text { Very High }\end{array}$ & Albumin $\left(p_{18}\right)$ & $\begin{array}{c}{[2.1-} \\
6.4]\end{array}$ & $\begin{array}{l}2.1-3.5 \text {; Low } \\
3.6-4.8 ; \\
\text { M e d i u m } \\
4.9-6.4 \text {; High }\end{array}$ \\
\hline Protime $\left(p_{19}\right)$ & $\begin{array}{lll}{\left[\begin{array}{lll}0 & & - \\
1 & 0 & 0\end{array}\right]} & -\end{array}$ & $\begin{array}{l}0-39 ; \text { Low } \\
39.1-58 ; \\
\text { M d i u m } \\
58.1-78 ; \text { High } \\
78.1-100 ; \\
\text { Very high }\end{array}$ & Hepatitis $\left(p_{d}\right)$ & -- & $\begin{array}{ll}\text { D i e } & \left(\begin{array}{l}0 \\
\text { L i ve }\end{array}\right. \\
(1)\end{array}$ \\
\hline
\end{tabular}

consolidated result of precision, recall, $\mathrm{F}$ - measure, and accuracy is presented in Table 8 . For better visualization, Figure 6 depicts the various measures of 5 folders.

\subsection{Comparative Analysis}

In this section, we compare our results with traditional rough set (Pawlak, 1982) model. The training dataset of 175 objects are analyzed using rough set with all features of the information system. The total number of rules generated using rough set is 37 . For better understanding the rules generated using rough set is presented in Table 9. The proposed ABCRS hybridized technique generates 19 which is $48.6 \%$ less than the total number of rules generated using rough set. 
Table 4. Sample information system of hepatitis disease

\begin{tabular}{|c|c|c|c|c|c|c|c|c|c|c|c|c|c|c|c|c|c|c|c|c|}
\hline Objects & $p_{1}$ & $p_{2}$ & $p_{3}$ & $p_{4}$ & $p_{5}$ & $p_{6}$ & $p_{7}$ & $p_{8}$ & $p_{9}$ & $p_{10}$ & $p_{11}$ & $p_{12}$ & $p_{13}$ & $p_{14}$ & $p_{15}$ & $p_{16}$ & $p_{17}$ & $p_{18}$ & $p_{19}$ & $p_{d}$ \\
\hline$q_{1}$ & 1 & 1 & 1 & 1 & 1 & 0 & 0 & 1 & 0 & 0 & 0 & 0 & 0 & 0 & 1 & 1 & 1 & 2 & 3 & 1 \\
\hline$q_{2}$ & 1 & 2 & 1 & 0 & 1 & 1 & 1 & 0 & 1 & 1 & 1 & 0 & 0 & 0 & 1 & 2 & 1 & 2 & 1 & 1 \\
\hline$q_{3}$ & 2 & 2 & 0 & 1 & 1 & 1 & 0 & 1 & 1 & 0 & 0 & 0 & 0 & 0 & 2 & 4 & 1 & 2 & 2 & 0 \\
\hline$q_{4}$ & 2 & 2 & 1 & 0 & 1 & 0 & 0 & 1 & 0 & 1 & 1 & 1 & 0 & 1 & 1 & 2 & 1 & 1 & 2 & 0 \\
\hline$q_{5}$ & 3 & 1 & 0 & 0 & 1 & 0 & 0 & 1 & 0 & 1 & 1 & 0 & 1 & 1 & 1 & 1 & 1 & 2 & 2 & 1 \\
\hline$q_{6}$ & 4 & 1 & 1 & 0 & 1 & 1 & 0 & 1 & 1 & 0 & 1 & 0 & 0 & 0 & 1 & 2 & 2 & 2 & 2 & 1 \\
\hline$q_{7}$ & 3 & 2 & 1 & 0 & 1 & 0 & 0 & 1 & 1 & 1 & 0 & 1 & 1 & 1 & 2 & 3 & 1 & 1 & 1 & 0 \\
\hline$q_{8}$ & 3 & 2 & 0 & 0 & 1 & 1 & 0 & 1 & 0 & 0 & 1 & 1 & 0 & 1 & 3 & 1 & 1 & 1 & 1 & 0 \\
\hline$q_{9}$ & 3 & 2 & 0 & 0 & 1 & 1 & 0 & 1 & 1 & 1 & 1 & 0 & 0 & 1 & 1 & 2 & 2 & 2 & 1 & 0 \\
\hline$q_{10}$ & 3 & 1 & 1 & 0 & 1 & 0 & 0 & 1 & 1 & 0 & 1 & 0 & 0 & 0 & 1 & 3 & 1 & 1 & 1 & 1 \\
\hline
\end{tabular}

Figure 5. Significance of features of hepatitis and setting of trend line

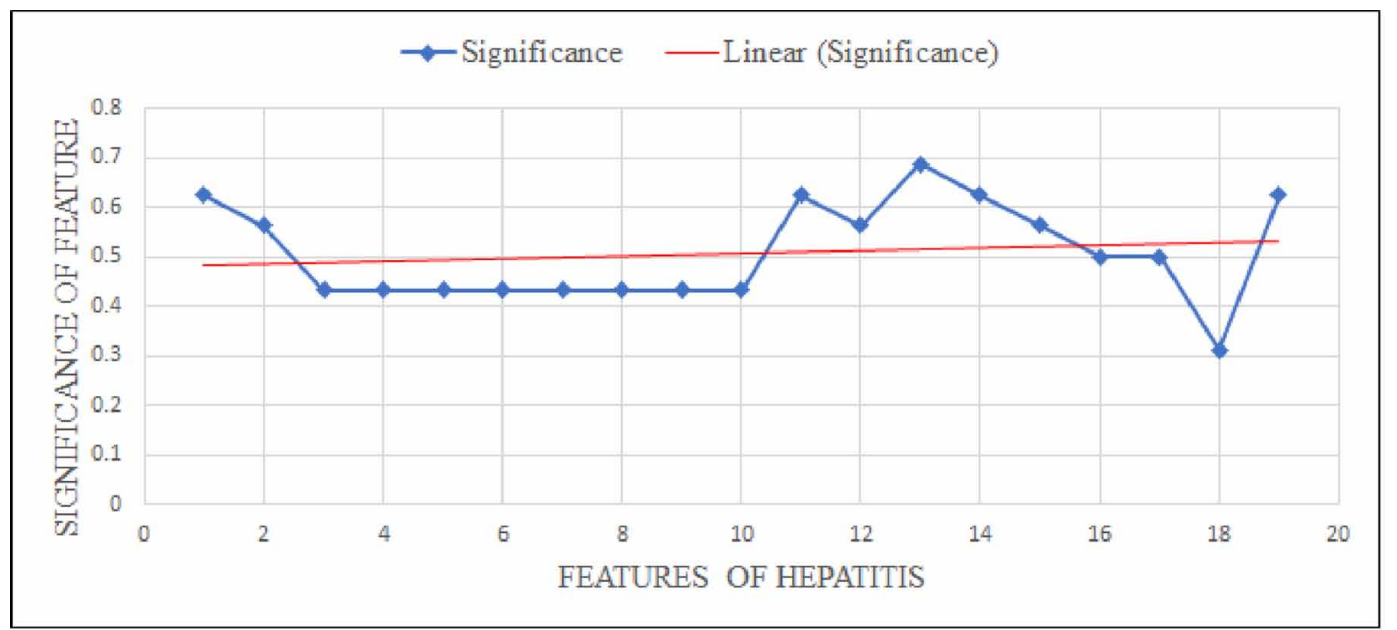


Table 5. Decision rules of hepatitis disease using proposed ABCRS hybridization

\begin{tabular}{|c|c|c|c|c|}
\hline Rule No. & Description & Support & Strength & Accuracy \\
\hline 1 & $\begin{array}{l}\text { If } p_{1}=\text { High, } p_{11}=\text { Yes, } p_{14}=\text { Yes, and } p_{19}= \\
\text { Low then } p_{d}=0\end{array}$ & 8 & $38.46 \%$ & $100 \%$ \\
\hline 2 & If $p_{11}=$ No and $p_{13}=$ Yes then $p_{d}=0$. & 5 & $15.38 \%$ & $100 \%$ \\
\hline 3 & $\begin{array}{l}\text { If } p_{1}=\text { Medium, } p_{12}=\text { Yes and } p_{15}=\text { Low then } \\
p_{d}=0 \text {. }\end{array}$ & 7 & $15.38 \%$ & $100 \%$ \\
\hline 4 & $\begin{array}{l}\text { If } p_{1}=\text { Medium, } p_{11}=\text { No, } p_{13}=\text { Yes and } p_{19}= \\
\text { High then } p_{d}=0 \text {. }\end{array}$ & 2 & $2.99 \%$ & $63 \%$ \\
\hline 5 & $\begin{array}{l}\text { If } p_{12}=\text { No, } p_{15}=\text { Medium and } p_{19}=\text { Medium } \\
\text { then } p_{d}=0\end{array}$ & 5 & $7.69 \%$ & $100 \%$ \\
\hline 6 & $\begin{array}{l}\text { If } p_{1}=\text { Medium, } p_{14}=\text { Yes and } p_{19}=\text { Low then } \\
p_{d}=0 \text {. }\end{array}$ & 7 & $15.38 \%$ & $100 \%$ \\
\hline 7 & If $p_{13}=$ Yes and $p_{19}=$ High then $p_{d}=0$. & 5 & $7.69 \%$ & $100 \%$ \\
\hline 8 & If $p_{13}=$ No and $p_{19}=$ High then $p_{d}=1$ & 23 & $31.34 \%$ & $100 \%$ \\
\hline 9 & $\begin{array}{l}\text { If } p_{12}=\text { No, } p_{15}=\text { Low and } p_{19}=\text { Medium then } \\
p_{d}=1\end{array}$ & 21 & $28.36 \%$ & $100 \%$ \\
\hline 10 & If $p_{1}=$ Low then $p_{d}=1$ & 5 & $7.46 \%$ & $100 \%$ \\
\hline 11 & If $p_{11}=$ Yes and $p_{19}=$ Very high then $p_{d}=1$ & 5 & $2.99 \%$ & $100 \%$ \\
\hline 12 & $\begin{array}{l}\text { If } p_{1}=\text { Low, } p_{12}=\text { Yes and } p_{15}=\text { Medium then } \\
p_{d}=1\end{array}$ & 5 & $1.49 \%$ & $55 \%$ \\
\hline 13 & If $p_{1}=$ Low and $p_{13}=$ Yes then $p_{d}=1$ & 3 & $1.49 \%$ & $57 \%$ \\
\hline 14 & If $p_{1}=$ Medium and $p_{13}=$ Yes then $p_{d}=1$ & 5 & $2.99 \%$ & $100 \%$ \\
\hline 15 & If $p_{12}=$ Yes and $p_{13}=$ Yes then $p_{d}=1$ & 3 & $1.49 \%$ & $100 \%$ \\
\hline
\end{tabular}


Table 5. Continued

\begin{tabular}{|c|l|c|c|c|}
\hline Rule No. & \multicolumn{1}{|c|}{ Description } & Support & Strength & Accuracy \\
\hline 16 & $\begin{array}{l}\text { If } p_{12}=\text { Yes, } p_{13}=\text { No and } p_{15}=\text { Medium then } \\
p_{d}=1\end{array}$ & 5 & $1.49 \%$ & $57 \%$ \\
\hline 17 & $\begin{array}{l}\text { If } p_{1}=\text { High, } p_{11}=\text { No and } p_{12}=\text { No then } \\
p_{d}=1\end{array}$ & 9 & $13.43 \%$ & $100 \%$ \\
\hline 18 & If $p_{14}=$ No and $p_{15}=$ Low then $p_{d}=1$. & 47 & $64.18 \%$ & $100 \%$ \\
\hline 19 & If $p_{12}=$ No and $p_{15}=$ Very high then $p_{d}=1$ & 5 & $1.49 \%$ & $100 \%$ \\
\hline
\end{tabular}

Table 6 Computation of accuracy for ABCRS hybridization model

\begin{tabular}{|c|c|c|c|c|c|c|c|c|}
\hline Description & $T P$ & $F N$ & $F P$ & $T N$ & Prec. & Rec. & F-Measure & Acc. \\
\hline Hepatitis = Live $p_{d}=1$ & 64 & 1 & 4 & 75 & 0.941 & 0.984 & 0.962 & $96.5 \%$ \\
\hline Hepatitis = Die $p_{d}=0$ & 69 & 2 & 4 & 69 & 0.945 & 0.972 & 0.958 & $95.8 \%$ \\
\hline Total & 133 & 3 & 8 & 144 & 0.943 & 0.978 & 0.960 & $96.2 \%$ \\
\hline
\end{tabular}

Table 7. Accuracy of ABCRS Model of Folder 1

\begin{tabular}{|c|c|c|c|c|c|c|c|c|}
\hline Description & $T P$ & $F N$ & $F P$ & $T N$ & Prec. & Rec. & F-Measure & Acc. \\
\hline Hepatitis = Live $p_{d}=1$ & 13 & 0 & 1 & 15 & 0.928 & 1.000 & 0.962 & $96.5 \%$ \\
\hline Hepatitis = Die $p_{d}=0$ & 14 & 0 & 1 & 14 & 0.933 & 1.000 & 0.965 & $96.5 \%$ \\
\hline Total & 27 & 0 & 2 & 29 & 0.931 & 1.000 & 0.964 & $96.5 \%$ \\
\hline
\end{tabular}

Table 8. Consolidated Measures of all Folders According to ABCRS Model

\begin{tabular}{|c|c|c|c|c|c|c|c|c|}
\hline Model & $T P$ & $F N$ & $F P$ & $T N$ & Prec. & Rec. & F-Measure & Acc. \\
\hline 1 & 27 & 0 & 2 & 29 & 0.931 & 1.000 & 0.964 & $96.5 \%$ \\
\hline 2 & 27 & 1 & 1 & 29 & 0.964 & 0.964 & 0.964 & $96.5 \%$ \\
\hline 3 & 27 & 0 & 2 & 29 & 0.931 & 1.000 & 0.964 & $96.5 \%$ \\
\hline 4 & 27 & 0 & 2 & 29 & 0.931 & 1.000 & 0.964 & $96.5 \%$ \\
\hline 5 & 25 & 2 & 1 & 28 & 0.961 & 0.925 & 0.943 & $94.6 \%$ \\
\hline
\end{tabular}


It is observed that, 4 rules out of 37 rules are having accuracy less than the predefined threshold of $65 \%$ and hence discarded. Based on the 33 rules, further on considering testing dataset of 144 objects, we have computed the confusion matrix for both the classes of hepatitis disease. The confusion matrix according to rough set model is presented in Table 10.

On comparing Table 6 and Table 8, we identified that ABCRS hybridization model gives accuracy of $96.2 \%$ whereas the rough set model gives accuracy of $92.0 \%$. For better visualization, precision, recall and accuracy of both the models is depicted in Figure 7. Thus, the hybridized ABCRS model gives better accuracy of $4.2 \%$ over rough set model.

\section{CONCLUSION}

Data are generated every moment at a higher speed. Additionally, it contains uncertainties. Analyzing those data to get some meaningful information is challenging. To this end in this paper, we have hybridized artificial bee colony with rough set (ABCRS) for knowledge inferencing. The proposed model, ABCRS, is analyzed over hepatitis dataset and compared with traditional rough set model. The proposed model, ABCRS, gives accuracy of $96.5 \%$ and $95.8 \%$ for the class hepatitis - live and hepatitis - die respectively. In overall it gives accuracy of $96.2 \%$. At the same time rough set model gives accuracy of $92.4 \%$ and $91.6 \%$ for the class hepatitis - live and hepatitis - die respectively. The overall accuracy of rough set model is $92 \%$. Therefore, it is clear that the proposed model ABCRS has given $4.2 \%$ better accuracy than the rough set model. Besides the proposed model, ABCRS, generates only 19 rules during rule generation phase whereas rough set generated 37 rules during rule generation phase. It indicates that, the proposed model, ABCRS, minimizes the rule generation procedure. It generates $48.6 \%$ less rules than traditional rough set model. While analyzing in real scenario, it is a great help to the physicians to take right decisions. The proposed model hybridizes rough set and artificial bee colony for feature selection and rule generation. The proposed work can be extended to fuzzy rough hybridization of artificial bee colony. Similarly, rough set can also be hybridized to other bio-inspired computing techniques. 
International Journal of Healthcare Information Systems and Informatics

Volume 16 • Issue 2 • April-June 2021

Figure 6. Various measures of all folders according to ABCRS model

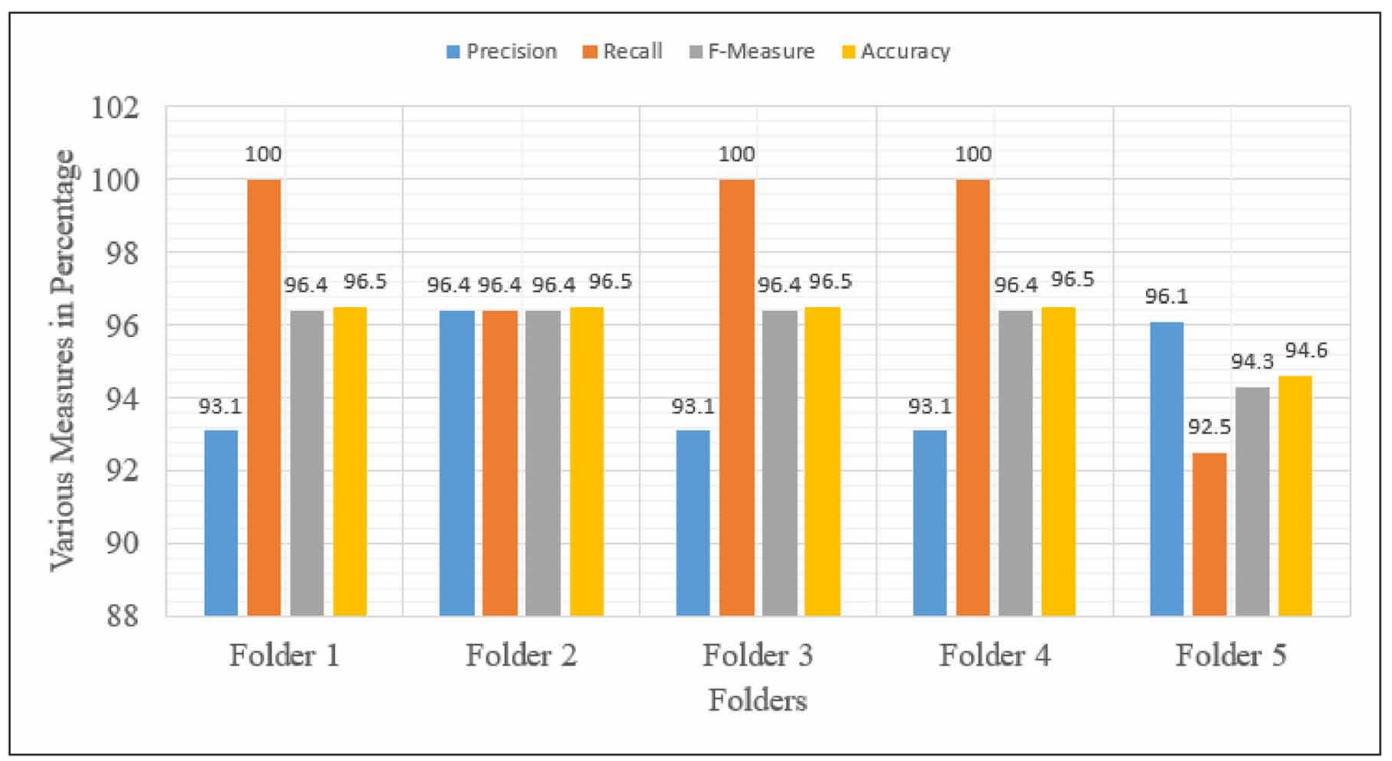


Table 9. Decision rules of hepatitis disease using proposed rough set model

\begin{tabular}{|c|c|c|c|c|}
\hline $\begin{array}{l}\text { Rule } \\
\text { No. }\end{array}$ & Description & Support & Strength & Accuracy \\
\hline 1 & If $p_{1}=$ High, $p_{3}=$ Yes, and $p_{13}=$ Yes then $p_{d}=0$ & 4 & $30.7 \%$ & $100 \%$ \\
\hline 2 & If $p_{1}=$ High, $p_{6}=$ Yes, and $p_{19}=$ Low then $p_{d}=0$ & 5 & $38.4 \%$ & $100 \%$ \\
\hline 3 & If $p_{1}=$ High, $p_{13}=$ Yes, and $p_{18}=$ Low then $p_{d}=0$ & 4 & $30.7 \%$ & $100 \%$ \\
\hline 4 & If $p_{5}=$ Yes, $p_{16}=$ Yes, and $p_{19}=$ Low then $p_{d}=0$ & 4 & $30.7 \%$ & $100 \%$ \\
\hline 5 & If $p_{6}=$ Yes, $p_{7}=$ No, and $p_{19}=$ Low then $p_{d}=0$ & 7 & $53.8 \%$ & $100 \%$ \\
\hline 6 & If $p_{6}=$ Yes, $p_{16}=$ Yes, and $p_{19}=$ Low then $p_{d}=0$ & 4 & $30.7 \%$ & $100 \%$ \\
\hline 7 & If $p_{7}=$ No, $p_{12}=$ Yes, and $p_{19}=$ Low then $p_{d}=0$ & 5 & $38.4 \%$ & $100 \%$ \\
\hline 8 & If $p_{8}=$ Yes, $p_{12}=$ Yes, and $p_{13}=$ Yes then $p_{d}=0$ & 4 & $30.7 \%$ & $100 \%$ \\
\hline 9 & If $p_{9}=$ Yes, $p_{19}=$ Low, and $p_{14}=$ Yes $\mathrm{t}$ hen $p_{d}=0$ & 4 & $30.7 \%$ & $100 \%$ \\
\hline 10 & If $p_{12}=$ Yes, $p_{13}=$ Yes, and $p_{18}=$ Low then $p_{d}=0$ & 4 & $30.7 \%$ & $100 \%$ \\
\hline 11 & If $p_{12}=$ Yes, $p_{17}=$ Yes, and $p_{19}=$ Low then $p_{d}=0$ & 4 & $30.7 \%$ & $100 \%$ \\
\hline 12 & If $p_{3}=$ Yes, and $p_{14}=$ No then $p_{d}=0$ & 7 & $40.3 \%$ & $100 \%$ \\
\hline 13 & If $p_{4}=$ No, and $p_{14}=$ No then $p_{d}=1$ & 3 & $46.2 \%$ & $100 \%$ \\
\hline 14 & If $p_{6}=$ No, and $p_{14}=$ No then $p_{d}=1$ & 4 & $47.7 \%$ & $100 \%$ \\
\hline 15 & If $p_{9}=$ No, and $p_{14}=$ No then $p_{d}=1$ & 8 & $41.7 \%$ & $100 \%$ \\
\hline 16 & If $p_{13}=$ No, and $p_{19}=$ High then $p_{d}=1$ & 3 & $31.3 \%$ & $65.00 \%$ \\
\hline 17 & If $p_{15}=$ Yes, and $p_{14}=$ No then $p_{d}=1$ & 4 & $64.1 \%$ & $100 \%$ \\
\hline 18 & If $p_{16}=$ Yes, and $p_{14}=$ No then $p_{d}=1$ & 5 & $49.5 \%$ & $100 \%$ \\
\hline 19 & If $p_{1}=$ Medium, $p_{3}=$ Yes, and $p_{10}=$ No then $p_{d}=1$ & 4 & $34.3 \%$ & $100 \%$ \\
\hline 20 & If $p_{1}=$ Medium, $p_{3}=$ Yes, and $p_{12}=$ No then $p_{d}=1$ & 5 & $37.3 \%$ & $100 \%$ \\
\hline
\end{tabular}


Table 9. Continued

\begin{tabular}{|c|c|c|c|c|}
\hline $\begin{array}{l}\text { Rule } \\
\text { No. }\end{array}$ & Description & Support & Strength & Accuracy \\
\hline 21 & If $p_{1}=$ Medium, $p_{6}=$ No, and $p_{16}=$ Yes then $p_{d}=1$ & 4 & $35.8 \%$ & $65 \%$ \\
\hline 22 & If $p_{3}=$ Yes, $p_{10}=$ No, and $p_{13}=$ No then $p_{d}=1$ & 8 & $43.2 \%$ & $100 \%$ \\
\hline 23 & If $p_{3}=$ Yes, $p_{11}=$ No, and $p_{12}=$ No then $p_{d}=1$ & 6 & $38.8 \%$ & $100 \%$ \\
\hline 24 & If $p_{3}=$ Yes, $p_{11}=$ No, and $p_{13}=$ No then $p_{d}=1$ & 6 & $38.8 \%$ & $100 \%$ \\
\hline 25 & If $p_{3}=$ Yes, $p_{12}=$ No, and $p_{13}=$ No then $p_{d}=1$ & 3 & $49.2 \%$ & $100 \%$ \\
\hline 26 & If $p_{3}=$ Yes, $p_{12}=$ No, and $p_{15}=$ Yes then $p_{d}=1$ & 4 & $49.2 \%$ & $100 \%$ \\
\hline 27 & If $p_{3}=$ Yes, $p_{13}=$ No, and $p_{16}=$ Yes then $p_{d}=1$ & 3 & $32.8 \%$ & $65 \%$ \\
\hline 28 & If $p_{3}=$ Yes,,$p_{13}=$ No, and $p_{18}=$ Medium then $p_{d}=1$ & 4 & $32.8 \%$ & $100 \%$ \\
\hline 29 & If $p_{3}=$ Yes, $p_{15}=$ Low, and $p_{18}=$ Medium then $p_{d}=1$ & 4 & $34.3 \%$ & $100 \%$ \\
\hline 30 & If $p_{6}=$ No, $p_{9}=$ No, and $p_{11}=$ No then $p_{d}=1$ & 4 & $35.8 \%$ & $100 \%$ \\
\hline 31 & If $p_{6}=$ No,,$p_{9=\text { No, }} p_{11=\text { No and }} p_{16}=$ Low then $p_{d}=1$ & 8 & $41.7 \%$ & $100 \%$ \\
\hline 32 & $\begin{array}{l}\text { If } \begin{array}{l}p_{6}=\text { No, } p_{13}=\text { No, } p_{11}=\text { No and } p_{16}=\text { Low then } \\
p_{d}=1\end{array}\end{array}$ & 4 & $46.2 \%$ & $100 \%$ \\
\hline 33 & If $p_{6}=$ No, $p_{15}=$ Low, and $p_{16}=$ Low then $p_{d}=1$ & 4 & $46.2 \%$ & $100 \%$ \\
\hline 34 & If $p_{9=\mathrm{No},} p_{11=\mathrm{No}, \text { and }} p_{12}=$ No then $p_{d}=1$ & 5 & $44.7 \%$ & $100 \%$ \\
\hline 35 & If $p_{9=\mathrm{No},} p_{11=\text { No, and }} p_{18}=$ Medium then $p_{d}=1$ & 4 & $32.8 \%$ & $65 \%$ \\
\hline 36 & If $p_{11=\text { No, }} p_{12}=$ No, and $p_{16}=$ Low then $p_{d}=1$ & 6 & $53.7 \%$ & $100 \%$ \\
\hline 37 & If $p_{11=\text { No, }} p_{16}=$ Low, and $p_{18}=$ Medium then $p_{d}=1$ & 6 & $38.8 \%$ & $100 \%$ \\
\hline
\end{tabular}


Table 10. Computation of accuracy for RS model

\begin{tabular}{|l|c|c|c|c|c|c|c|c|}
\hline Description & $T P$ & $F N$ & $F P$ & $T N$ & Prec. & Rec. & F-Measure & Acc. \\
\hline Hepatitis = Live $p_{d}=1$ & 58 & 4 & 7 & 75 & 0.892 & 0.935 & 0.913 & $92.4 \%$ \\
\hline Hepatitis = Die $p_{d}=0$ & 63 & 3 & 9 & 69 & 0.875 & 0.954 & 0.912 & $91.6 \%$ \\
\hline Total & 121 & 7 & 16 & 144 & 0.883 & 0.945 & 0.913 & $92.0 \%$ \\
\hline
\end{tabular}

Figure 7. Various measures of Both ABCRS and RS model

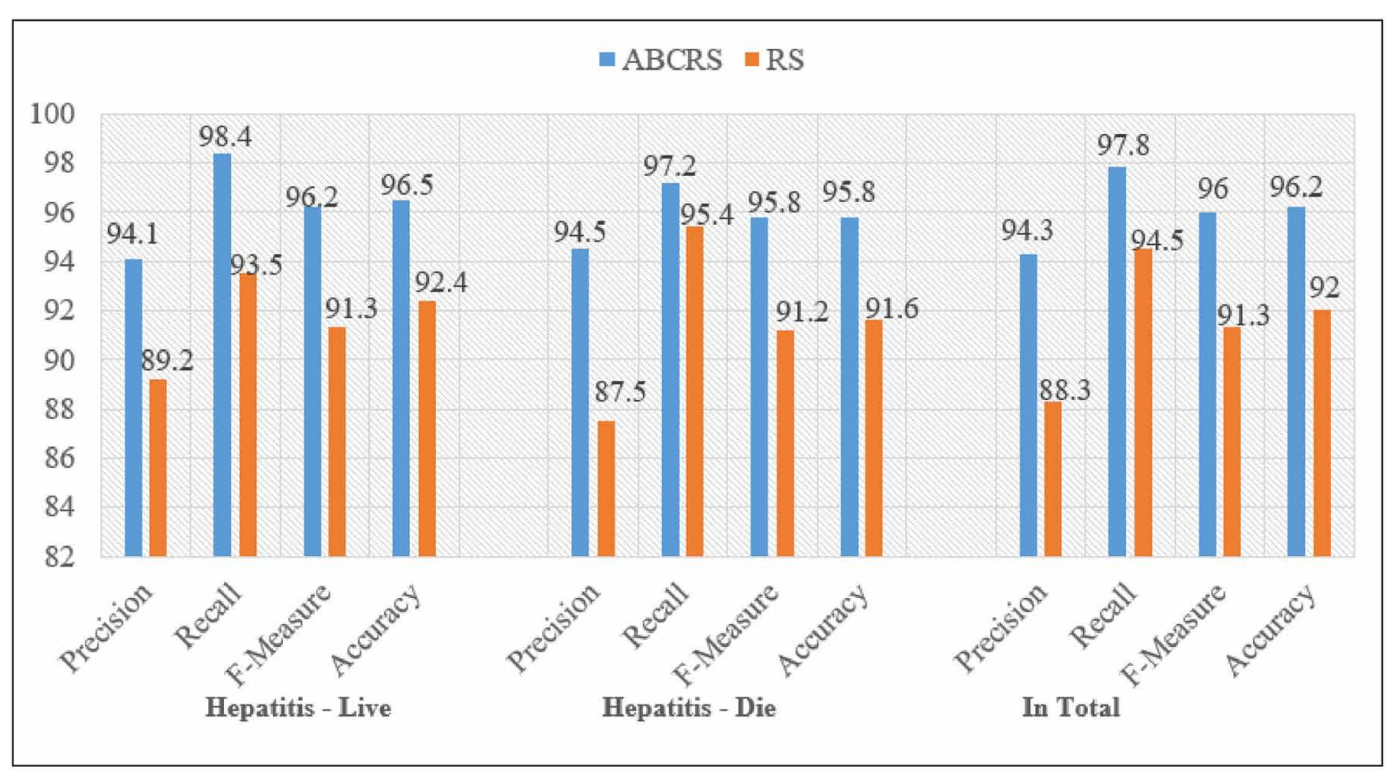




\section{REFERENCES}

Acharjya, D. P., \& Tripathy, B. K. (2007). Rough Sets on Intuitionistic. Fuzzy Approximation Spaces and Knowledge Representation. Journal, Elsevier, 177(1), 28-40.

Acharjya, D. P., \& Tripathy, B. K. (2008). Rough sets on fuzzy approximation spaces and applications to distributed knowledge systems. International Journal of Artificial Intelligence and Soft Computing, 1(1), 1-14. doi:10.1504/IJAISC.2008.021260

Acharjya, D. P., \& Tripathy, B. K. (2012). Intuitionistic fuzzy rough set on two universal sets and knowledge representation. Mathematical Sciences International Research Journal, 1(2), 584-598.

Awadallah, M. A., Al-Betar, M. A., Bolaji, A. L. A., Alsukhni, E. M., \& Al-Zoubi, H. (2019). Natural selection methods for artificial bee colony with new versions of onlooker bee. Soft Computing, 23(15), 6455-6494. doi:10.1007/s00500-018-3299-2

Bao, L., \& Zeng, J. C. (2009). Comparison and analysis of the selection mechanism in the artificial bee colony algorithm. In Hybrid Intelligent Systems, 2009. HIS'09. Ninth International Conference on (Vol. 1, pp. 411416). IEEE.

Bin Basir, M. A., \& binti Ahmad, F. (2014). Comparison on Swarm Algorithms for Feature Selections/Reductions. International Journal of Science and Engineering.

Das, T. K., \& Acharjya, D. P. (2014). A decision making model using soft set and rough set on fuzzy approximation spaces. International Journal of Intelligent Systems Technologies and Applications, 13(3), 170-186. doi:10.1504/ IJISTA.2014.065172

Dash, M., \& Liu, H. (1997). Feature selection for classification. Intelligent Data Analysis, 1(3), $131-156$. doi:10.3233/IDA-1997-1302

Dubois, D., \& Prade, H. (1990). Rough fuzzy sets and fuzzy rough sets. International Journal of General Systems, 17(2-3), 191-209. doi:10.1080/03081079008935107

Forsati, R., Moayedikia, A., \& Keikha, A. (2012). A novel approach for feature selection based on the bee colony optimization. International Journal of Computers and Applications, 43(8), 30-34.

Guyon, I., \& Elisseeff, A. (2003). An introduction to variable and feature selection. Journal of Machine Learning Research, 3(Mar), 1157-1182.

Hancer, E., Xue, B., Zhang, M., Karaboga, D., \& Akay, B. (2018). Pareto front feature selection based on artificial bee colony optimization. Information Sciences, 422, 462-479. doi:10.1016/j.ins.2017.09.028

Jain, A., \& Zongker, D. (1997). Feature selection: Evaluation, application, and small sample performance. IEEE Transactions on Pattern Analysis and Machine Intelligence, 19(2), 153-158. doi:10.1109/34.574797

Karaboga, D., \& Akay, B. (2009). A comparative study of artificial bee colony algorithm. Applied Mathematics and Computation, 214(1), 108-132. doi:10.1016/j.amc.2009.03.090

Karaboga, D., \& Basturk, B. (2008). On the performance of artificial bee colony (ABC) algorithm. Applied Soft Computing, 8(1), 687-697. doi:10.1016/j.asoc.2007.05.007

Karaboga, D., \& Ozturk, C. (2010). Fuzzy clustering with artificial bee colony algorithm. Scientific Research and Essays, 5(14), 1899-1902.

Karaboga, D., \& Ozturk, C. (2011). A novel clustering approach: Artificial Bee Colony (ABC) algorithm. Applied Soft Computing, 11(1), 652-657. doi:10.1016/j.asoc.2009.12.025

Kaur, A., \& Goyal, S. (2011). A survey on the applications of bee colony optimization techniques. International Journal on Computer Science and Engineering, 3(8), 3037.

Kohavi, R., \& John, G. H. (1997). Wrappers for feature subset selection. Artificial Intelligence, 97(1-2), $273-324$. doi:10.1016/S0004-3702(97)00043-X

Palanisamy, S., \& Kanmani, S. (2012). Artificial bee colony approach for optimizing feature selection. International Journal of Computer Science Issues, 9(3), 432. 
Pawlak, Z. (1982). Rough sets. International Journal of Computer \& Information Sciences, 11(5), 341-356.

Rao, H., Shi, X., Rodrigue, A. K., Feng, J., Xia, Y., Elhoseny, M., Yuan, X., \& Gu, L. (2019). Feature selection based on artificial bee colony and gradient boosting decision tree. Applied Soft Computing, 74, 634-642. doi:10.1016/j.asoc.2018.10.036

Rathee, G., Sharma, A., Saini, H., Kumar, R., \& Iqbal, R. (2019). A hybrid framework for multimedia data processing in IoT-healthcare using blockchain technology. Multimedia Tools and Applications. Advance online publication. doi:10.1007/s11042-019-07835-3

Saxena, A., Gavel, L. K., \& Shrivas, M. M. (2014). Rough sets for feature selection and classification: An overview with applications. International Journal of Recent Technology and Engineering, 3(5), 62-69.

Schiezaro, M., \& Pedrini, H. (2013). Data feature selection based on Artificial Bee Colony algorithm. EURASIP Journal on Image and Video Processing, 2013(1), 47. doi:10.1186/1687-5281-2013-47

Sharma, A., \& Kumar, R. (2019a). A constrained framework for context-aware remote E-healthcare (CARE) services. Transactions on Emerging Telecommunications Technologies. Advance online publication. doi:10.1002/ ett.3649

Sharma, A., \& Kumar, R. (2019b). Service level agreement and energy cooperative cyber physical system for quickest healthcare services. Journal of Intelligent \& Fuzzy Systems, 36(5), 4077-4089. doi:10.3233/JIFS-169968

Sharma, A., \& Kumar, R. (2019c). Computation of the reliable and quickest data path for healthcare services by using service-level agreements and energy constraints. Arabian Journal for Science and Engineering, 44(11), 9087-9104. doi:10.1007/s13369-019-03836-4

Sharma, A., Rathee, G., Kumar, R., Saini, H., Vijaykumar, V., Nam, Y., \& Chilamkurti, N. (2019). A Secure, Energy-and SLA-Efficient (SESE) E-Healthcare Framework for Quickest Data Transmission Using CyberPhysical System. Sensors (Basel), 19(9), 1-22. doi:10.3390/s19092119 PMID:31067811

Shokouhifar, M., \& Sabet, S. (2010). A hybrid approach for effective feature selection using neural networks and artificial bee colony optimization. 3rd International Conference on Machine Vision (ICMV 2010), 502-506.

Shu, W., \& Shen, H. (2014). Incremental feature selection based on rough set in dynamic incomplete data. Pattern Recognition, 47(12), 3890-3906. doi:10.1016/j.patcog.2014.06.002

Swiniarski, R. W., \& Skowron, A. (2003). Rough set methods in feature selection and recognition. Pattern Recognition Letters, 24(6), 833-849. doi:10.1016/S0167-8655(02)00196-4

Tripathy, B. K., \& Acharjya, D. P. (2013). Approximation of classification and measures of uncertainty in rough set on two universal sets. arXiv preprint arXiv:1301.6789

Tripathy, B. K., Acharjya, D. P., \& Cynthya, V. (2013). A framework for intelligent medical diagnosis using rough set with formal concept analysis. arXiv preprint arXiv:1301.6011

Uzer, M. S., Yilmaz, N., \& Inan, O. (2014). A Case Study: Effect of ABC-based Feature Selection Algorithm on Breast Cancer Diagnosis. Global Journal on Technology, 5, 163-169.

Wang, S., \& Dong, R. (2019). Feature selection with improved binary artificial bee colony algorithm for microarray data. International Journal on Computer Science and Engineering, 19(3), 387-399.

Xiao, S., Wang, W., Wang, H., Tan, D., Wang, Y., Yu, X., \& Wu, R. (2019). An Improved Artificial Bee Colony Algorithm Based on Elite Strategy and Dimension Learning. Mathematics, 7(3), 289. doi:10.3390/math7030289

Zhong, N., Dong, J., \& Ohsuga, S. (2001). Using rough sets with heuristics for feature selection. Journal of Intelligent Information Systems, 16(3), 199-214. doi:10.1023/A:1011219601502 
Kauser Ahmed P. received his M. Tech. (IT) with specialization in networking; M. Sc. (Computer Science) from VIT University, Vellore, India and currently pursuing his PhD in Computer Science and Engineering from VIT, Vellore, India. Presently he is working as an Assistant Professor in the school of computing sciences and engineering, VIT University, Vellore, India. He has published several peer-reviewed national and international conference and journal papers. He is associated with many professional bodies like CSI, ISTE, IACSIT, CSTA, IEEE, IRSS and IAENG. He is a reviewer of many international conferences. His current research interests include soft computing, big data analytics, bio-inspired computing, and bio informatics.

D. P. Acharjya received his PhD in computer science from Berhampur University, India. He has been awarded with Gold Medal in M. Sc. from NIT, Rourkela. Currently he is working as a Professor in the School of Computing Science and Engineering, VIT University, Vellore, India. He has authored many national and international journal papers, book chapters, and five books to his credit. Additionally he has edited seven books to his credit. He is reviewer of many international journals such as Fuzzy Sets and Systems, Knowledge Based Systems, and Applied Soft Computing. He has been awarded with Eminent Academician Award from Khallikote Sanskrutika Parisad, Berhampur, Odisha; and Outstanding Educator and Scholar Award from National Foundation for Entrepreneurship Development, Coimbatore; and Bharat Vikas Award from Institute of Self Reliance, Bhubaneswar, Odisha. Dr. Acharjya is actively associated with many professional bodies like CSI, ISTE, IMS, AMTI, ISIAM, OITS, IACSIT, CSTA, IEEE and IAENG. He was founder secretary of OITS Rourkela chapter. His current research interests include rough sets, formal concept analysis, knowledge representation, data mining, granular computing, bioinspired computing, and business intelligence. 